\title{
Cuando el coito produce dolor: una exploración de la sexualidad femenina en el noroeste de México
}

\author{
Hilda García-Pérez, PhD, (I) Sioban D Harlow, PhD ${ }^{(2)}$
}

\begin{abstract}
García-Pérez H, Harlow SD.
Cuando el coito produce dolor: una exploración de la sexualidad femenina en el noroeste de México. Salud Publica Mex 2010;52:148-I55.
\end{abstract}

\begin{abstract}
Resumen
Objetivo. Determinar la prevalencia de dispareunia y los factores de riesgo asociados en población femenina en edades de 25 a 54 años. Material y métodos. Se analizó información de I 183 mujeres sexualmente activas que participaron en un estudio poblacional de tipo transversal en la ciudad de Hermosillo, Sonora. La asociación entre dispareunia y variables sociodemográficas, antecedentes médicos y violencia sexual se analizó usando regresión logística múltiple. Resultados. La prevalencia de dispareunia durante el año previo a la encuesta fue de $12.3 \%$ (IC $95 \%$ I0.5-I4.4\%). Después de controlar por la ocupación, se encontró que la dispareunia estuvo asociada con mujeres jóvenes (25-34 años), antecedentes de enfermedades de transmisión sexual y/o enfermedad inflamatoria de la pelvis, infección urinaria crónica, colitis y violencia sexual. Conclusión. Es urgente incrementar el escrutinio y la atención de la dispareunia en el contexto de los programas nacionales de salud sexual y reproductiva y en los servicios de atención primaria a la salud.
\end{abstract}

Palabras clave: dispareunia; relaciones sexuales; sexualidad; violencia sexual; salud de las mujeres; México
García-Pérez H, Harlow SD.

When coitus produce pain: an exploration of female sexuality in northwest Mexico.

Salud Publica Mex 2010;52:148-I55.

\section{Abstract}

Objective. To determine the prevalence of dyspareunia among women aged 25-54 and its associated risk factors. Material and methods. A cross-sectional populationbased study was carried out in the city of Hermosillo, Sonora and data from I I 83 sexually active women were analyzed. A multiple logistic regression was computed to analyze the association between dyspareunia and sociodemographic characteristics, medical conditions and sexual violence. Results. The 12-month prevalence of dyspareunia was estimated to be $12.3 \%(95 \% \mathrm{Cl} 10.5,14.4)$. After adjustment for working conditions, dyspareunia was associated with younger ages (25-34 years), history of sexually transmitted diseases/pelvic inflammatory disease, chronic urinary tract infections, colitis and history of sexual violence. Conclusion. Increased attention to this condition by reproductive health programs and primary care services is urgently needed in Mexico.

Key words: dyspareunia; sexual intercourse; sexuality; women's sexual violence; health; Mexico

Esta investigación fue financiada por The Maternal and Child Health Research Training Program de Fogarty International Center, National Institutes of Health (D43 TW01276), por la Fundación Ford a través del proyecto Construyendo nuevos espacios para género y salud en el noroeste de México (Grant No. I000124I) del programa Salud y Sociedad del Colegio de Sonora y el Block Grant de Rackham Graduate School de la Universidad de Michigan en Ann Arbor.

(I) Departamento de Estudios Transfronterizos de Chicanas/os y Latinas/os, Universidad Estatal de Arizona.Arizona, Estados Unidos.

(2) Departamento de Epidemiología, Escuela de Salud Pública, Universidad de Michigan. Michigan, Estados Unidos.

Fecha de recibido: 4 de mayo de 2009 - Fecha de aceptado: 16 de octubre de 2009

Solicitud de sobretiros: Dra. Hilda García Pérez. Departamento de Estudios Transfronterizos de Chicanas/os y Latinas/os,

Universidad Estatal de Arizona. PO Box 873502, Tempe Arizona 85287-3502. Estados Unidos. Correo electrónico: hilda.garcia@asu.edu 
A pesar de los avances realizados desde la adopción del concepto de salud reproductiva en la década de los noventa, los principios que rigen las políticas de salud en esta área continúan dando énfasis en la actualidad en los procesos de enfermedad y de salud y refuerzan una visión muy limitada de las necesidades de salud de la población femenina del país. ${ }^{1}$ Aunque el uso del eslogan "sexo seguro" es recurrente en algunos de los programas de salud reproductiva, es claro que la promoción del placer y la satisfacción sexual no son componentes explícitos de dichos programas, como tampoco lo es la prevención o evaluación de los trastornos sexuales.

Debido a que los programas de salud reproductiva en México manifiestan preocupación por enfermedades que ponen en riesgo la vida de las mujeres, los trastornos sexuales han sido tradicionalmente relegados de la investigación y de los programas de salud dirigidos a las mismas. ${ }^{1}$ Una revisión rápida de los programas de salud reproductiva muestran un énfasis en el área de la salud materno-infantil, planificación familiar, enfermedades de transmisión sexual y cánceres ginecológicos. ${ }^{2,3}$ Como resultado de esto, la información disponible sobre trastornos sexuales femeninos es muy limitada ${ }^{4-6}$ y la investigación sobre el efecto de estos desórdenes en la calidad de vida de las mujeres es virtualmente inexistente. ${ }^{7}$ Esta situación es inaceptable desde una perspectiva de salud pública ya que estudios realizados en otros países indican que los desórdenes sexuales como la dispareunia o el coito doloroso son dos de los trastornos sexuales más comunes en la población femenina ${ }^{8}$ con repercusiones importantes en la calidad de vida de esta población. ${ }^{7,9}$

Aunque la dispareunia es una condición que afecta a hombres y mujeres, ésta tiende a ser más prevalente en población femenina. ${ }^{10,11}$ La dispareunia es el dolor que se experimenta en los genitales y la estructura pélvica durante la relación sexual o durante las 24 horas posteriores al coito. ${ }^{10,12}$ En mujeres en edad reproductiva la prevalencia de dispareunia se encuentra en el rango de 10 a 15\% 9,11 mientras que en mujeres en etapa premenopáusica el rango varía de entre 3 a 33\%. ${ }^{11,13}$ Aunque diversas morbilidades ginecológicas, condiciones médicas y trastornos psicológicos han sido identificadas como factores de riesgo para la dispareunia, ${ }^{8}$ algunos estudios relacionan esta condición con el abuso físico y sexual a mujeres así como con relaciones de pareja no satisfactorias. $^{8}$

Este estudio busca determinar la prevalencia de dispareunia o dolor pélvico durante el coito o 24 horas después del mismo en población femenina en edad reproductiva (25-54 años), residente del área urbana de
Hermosillo, Sonora. La severidad de dicho trastorno y los cambios en la frecuencia de las relaciones sexuales también son analizados. Asimismo, se evalúan los factores sociales y biomédicos asociados con dicho trastorno.

\section{Material y métodos}

Este trabajo es parte de un estudio más amplio sobre morbilidad ginecológica y calidad de vida en mujeres en edad reproductiva en el área urbana de Hermosillo, Sonora. ${ }^{7}$ En este estudio se analiza información de una muestra aleatoria de mujeres en edades de 25 a 54 años residentes en el área urbana de la ciudad de Hermosillo. La muestra se extrajo de un grupo de 29 áreas geoestadísticas básicas (AGEB) seleccionadas aleatoriamente de un listado de 234 AGEB con al menos 50 habitantes. El procedimiento de muestreo aleatorio se basó en la cartografía censal elaborada por el Instituto Nacional de Estadística Geografía e Informática durante el Censo de Población y Vivienda 2000. En cada AGEB seleccionada, se eligieron aleatoriamente diez manzanas, mismas que fueron encuestadas secuencialmente hasta alcanzar un promedio de 45 mujeres entrevistadas por AGEB. Para la recolección de los datos se usó un cuestionario filtro para identificar mujeres en edades de 25 a 54 años que fueran residentes permanentes de la ciudad de Hermosillo. Se entrevistó una mujer por hogar y en los casos de los hogares donde había más de una mujer en el rango de edad se usó un procedimiento aleatorio para seleccionar a la informante. Se identificaron 1420 mujeres de las cuales 1307 (92\%) fueron entrevistadas, $49(3.4 \%)$ declinaron participar y $64(4.5 \%)$ no pudieron ser contactadas. El protocolo del estudio fue aprobado por el Comité de ética de la Universidad de Michigan, El Colegio de Sonora y la Universidad Estatal de Arizona. Previo a la entrevista las participantes firmaron un consentimiento informado. Para la recolección de los datos se usó un cuestionario estructurado que fue aplicado por estudiantes de enfermería y por enfermeras de la Secretaría de Salud durante los meses de mayo a agosto de 2005.

\section{Dispareunia}

La dispareunia es el dolor que se experimenta durante la relación sexual o 24 horas después de ésta en un área que incluye los genitales y la estructura pélvica. ${ }^{10,12} \mathrm{La}$ dispareunia se evaluó en mujeres de 25-54 años que durante los 12 meses previos a la encuesta reportaron tener una pareja sexual $(\mathrm{n}=1$ 183). 


\section{Variables reproductivas y otras morbilidades}

La edad de inicio a la actividad sexual, el uso de un dispositivo intrauterino, los antecedentes de enfermedades de transmisión sexual, antecedentes de enfermedad inflamatoria pélvica, dolor crónico de la pelvis, antecedentes de colitis ulcerativa y otros trastornos intestinales, además de contar con un diagnóstico de infección crónica de las vías urinarias (infección recurrente de los riñones y / o cistitis crónica), integran las variables incluidas en este componente.

\section{Violencia sexual padecida recurrentemente durante toda la vida}

Se evaluó la violencia sexual de toda la vida de acuerdo a las preguntas de un cuestionario desarrollado por la Organización Mundial de la Salud (OMS) ${ }_{,}^{14}$ y a los siguiente criterios: a) si la violencia sexual ocurrió antes de los 15 años y si ésta fue perpetrada por alguien distinto a la pareja o cónyuge. Esta variable incluye la presencia de un coito forzado y / o el hecho de que la mujer haya sido "tocada" u obligada a hacer "algo sexual" en contra de su voluntad; b) si desde los 15 años alguien la forzó a tener relaciones sexuales o a realizar algún acto sexual en contra de su voluntad. Esta variable incluye la violencia sexual por parte de la pareja o cónyuge o la violencia sexual de alguien distinto al cónyuge; y c) si la violencia sexual es una combinación de varios eventos de violencia sexual en distintos momentos y por distintos perpetradores.

También se evaluó la violencia sexual ocurrida exclusivamente dentro del periodo en que la mujer ha vivido en una relación de pareja. Esta variable mide la prevalencia de los últimos 12 meses y la prevalencia de violencia sexual de toda la vida viviendo en pareja. El cuestionamiento sobre violencia de pareja incluyó tres preguntas: a) si la mujer fue forzada físicamente a tener relaciones sexuales cuando ella no lo deseaba; $b$ ) si tuvo relaciones sexuales con su cónyuge o pareja por temor o miedo y; c) si fue forzada a realizar algún acto sexual catalogado por ella de humillante o degradante.

\section{Variables sociodemográficas}

Las variables de control fueron edad, educación, estado civil y estatus ocupacional de las mujeres.

Se calcularon estadísticas descriptivas con SPSS 16.0 incluyendo frecuencias de enfermedad y medidas de tendencia central. Se calcularon razones de momios crudas y ajustadas para el incremento en la probabilidad de reporte de dispareunia. La dispareunia se analizó en su asociación con distintas variables usando de regresión logística múltiple. Se presentan los intervalos de confianza para un nivel de significancia del $95 \%$. Asimismo, se evaluó la posible colinealidad de las variables y la existencia de interacciones estadísticas.

\section{Resultados}

Durante los 12 meses previos a la encuesta, la prevalencia de dispareunia o dolor pélvico durante o 24 horas después del coito vaginal fue reportada por $12.3 \%$ (IC 95\% 10.5-14.4\%) de las participantes, mientras que la prevalencia de dicho trastorno el mes previo a la encuesta fue del 9.2\% (cuadro I).

El cuadro II muestra la distribución relativa de las mujeres en edad de 25 a 54 años, la prevalencia de dispareunia y las razones de momios crudas de acuerdo a variables sociodemográficas, morbilidad ginecológica, variables reproductivas y violencia sexual. Aproximadamente 1 de cada 3 mujeres participantes tenía entre 25 y 34 años, 27\% tenían educación primaria o menos y $41 \%$ había terminado o cursado por lo menos un año de educación secundaria. La gran mayoría de las participantes vivían en alguna forma de unión (81.3\%), aunque $63.9 \%$ reportaron estar casadas. Tres de cada 5 mujeres declaró como su actividad principal dedicarse al hogar.

De acuerdo con estos datos no se observó asociación significativa entre el reporte de dispareunia y las variables de educación y estado civil (cuadro II). Por otra parte, las mujeres adultas jóvenes (25-34 años) y las mujeres trabajadoras mostraron un mayor riesgo de dispareunia en comparación con mujeres adultas maduras (35-54 años) y las mujeres que se encontraban en el hogar. El conjunto de las variables de salud reproductiva incluidas en el análisis fueron significativas. La presencia de una patología crónica en el área pélvica

Cuadro I

Prevalencia de dispareunia durante los 12 meses $\mathrm{Y}$ LOS 30 DÍAS PREVIOS A LA ENCUESTA. $\mathrm{N}=\mathbf{I}$ | 83. ENCUESTA SOBRE MORBILIDAD GINECOLÓGICA Y CALIDAD DE VIDA EN MUJERES EN EDAD REPRODUCTIVA. Hermosillo, Sonora, 2005

\begin{tabular}{lccc}
\cline { 2 - 4 } Prevalencia de dispareunia & $n$ & $\%$ & IC 95\% \\
\hline 12 meses previos & 146 & 12.3 & $10.5 \quad$ ।4.4 \\
\hline 30 días previos & 109 & 9.2 & $7.7 \quad$ II.0 \\
\hline
\end{tabular}




\section{Cuadro II}

DISTRIBUCIÓN, PREVALENCIA Y RAZÓN DE MOMIOS DE LA ASOCIACIÓN ENTRE DISPAREUNIA Y VARIABLES SOCIODEMOGRÁFICAS, REPRODUCTIVAS, VIOLENCIA SEXUAL Y CONDICIONES MÉDICAS DIAGNOSTICADAS ( $N=\mid$ | 83). ENCUESTA SOBRE MORBILIDAD ginecológica y Calidad de vida en mujeres en edad Reproductiva. Hermosillo, Sonora, 2005

\begin{tabular}{|c|c|c|c|}
\hline Sociodemográficas & $\begin{array}{c}\text { Frecuencia } \\
n\end{array}$ & $\begin{array}{c}\text { Prevalencia } \\
n(\%)\end{array}$ & $\begin{array}{l}\text { Razón de momios } \\
\text { (IC } 95 \% \text { ) }\end{array}$ \\
\hline \multicolumn{4}{|l|}{ Grupos de edad } \\
\hline $25-34$ & $436(36.9)$ & $76(17.4)$ & $2.04(1.442 .89)$ \\
\hline $35-54$ & $747(76.1)$ & $70(9.37)$ & I.0 Referencia \\
\hline \multicolumn{4}{|l|}{ Educación } \\
\hline Ninguna/Primaria & $318(26.9)$ & $34(10.7)$ & $0.84(0.52 \quad 1.34)$ \\
\hline Secundaria & $487(4 I .2)$ & $65(13.3)$ & $1.08(0.72 \mathrm{I} .6 \mathrm{I})$ \\
\hline Preparatoria/universidad & $377(31.9)$ & $47(12.4)$ & 1.0 Referencia \\
\hline$N / R$ & 1 & & \\
\hline \multicolumn{4}{|l|}{ Estado civil } \\
\hline Soltera & $88(7.4)$ & $5(5.7)$ & $0.43(0.171 .08)$ \\
\hline Casada & $820(63.9)$ & $101(12.3)$ & 1.0 Referencia \\
\hline Unión libre & $206(17.4)$ & $29(14.1)$ & $1.16(0.74 \quad 1.82)$ \\
\hline Divorciada/separada/viuda & $69(5.8)$ & II (15.9) & $1.35(0.682 .65)$ \\
\hline \multicolumn{4}{|l|}{ Ocupación } \\
\hline Trabaja & $442(37.4)$ & $68(15.4)$ & $1.60(1.122 .28)$ \\
\hline Hogar & $717(60.6)$ & $73(10.2)$ & 1.0 Referencia \\
\hline Otro & $24(2.0)$ & $5(20.8)$ & $2.32(0.846 .40)$ \\
\hline \multicolumn{4}{|l|}{ Reproductivas } \\
\hline \multicolumn{4}{|l|}{ Edad de inicio de la actividad sexual } \\
\hline$<15$ & $5 \mathrm{I}(4.3)$ & $12(23.5)$ & $2.54(1.285 .02)$ \\
\hline $15-17$ & $279(23.6)$ & $42(15.1)$ & $1.46(0.982 .17)$ \\
\hline $18+$ & $852(73.1)$ & $92(10.8)$ & 1.0 Referencia \\
\hline$N / R$ & 1 & & \\
\hline \multicolumn{4}{|l|}{ Uso DIU } \\
\hline Sí & $110(9.3)$ & $22(20.0)$ & $1.91(1.163 .16)$ \\
\hline No & $1073(90.7)$ & $124(\mid 1.6)$ & 1.0 Referencia \\
\hline \multicolumn{4}{|l|}{ Antecedentes de ETS/EIP } \\
\hline Sí & $153(13.0)$ & $37(24.2)$ & $2.71(1.784 .13)$ \\
\hline No & $1028(87.0)$ & $108(10.5)$ & I.0 Referencia \\
\hline $\mathrm{N} / \mathrm{R}$ & 2 & & \\
\hline \multicolumn{4}{|l|}{ Dolor crónico de la pelvis } \\
\hline Sí & $123(10.4)$ & $57(46.3)$ & $9.4 \mid(6.2 \mid 14.26)$ \\
\hline No & $1059(89.6)$ & $89(8.4)$ & 1.0 Referencia \\
\hline $\mathrm{N} / \mathrm{R}$ & 1 & & \\
\hline \multicolumn{4}{|l|}{ Condiciones médicas } \\
\hline \multicolumn{4}{|l|}{ Colitis ulcerativa y otros trastornos intestinales } \\
\hline Sí & $393(33.3)$ & $70(17.8)$ & $2.08(1.462 .97)$ \\
\hline No & $787(66.7)$ & $74(9.4)$ & 1.0 Referencia \\
\hline $\mathrm{N} / \mathrm{R}$ & 3 & & \\
\hline \multicolumn{4}{|l|}{ Infección crónica de las vías urinarias } \\
\hline Sí & $483(40.9)$ & $82(17.0)$ & $2.06(1.452 .92)$ \\
\hline No & $698(59.1)$ & $63(9.0)$ & 1.0 Referencia \\
\hline $\mathrm{N} / \mathrm{R}$ & 2 & & \\
\hline Violencia & & & \\
\hline \multicolumn{4}{|l|}{ Violencia sexual de toda la vida } \\
\hline Sólo antes de los 15 años & $47(4.0)$ & $16(34.0)$ & $4.79(2.539 .08)$ \\
\hline Sólo a partir de los 15 años & $95(8.1)$ & $20(21.1)$ & $2.47(1.454 .23)$ \\
\hline Antes y después de los 15 años & $38(3.2)$ & $12(31.6)$ & $4.28(2.098 .76)$ \\
\hline No violencia & $999(84.7)$ & $97(12.3)$ & I.0 Referencia \\
\hline $\mathrm{N} / \mathrm{R}$ & 5 & & \\
\hline \multicolumn{4}{|l|}{ Violencia sexual de la pareja o cónyuge } \\
\hline Los pasados 12 meses & $37(3.1)$ & $10(27.0)$ & $2.83(1.346 .01)$ \\
\hline Antes de los 12 meses & $67(5.3)$ & $12(19.0)$ & $1.80(0.933 .47)$ \\
\hline No aplica/ no respuesta & $52(4.4)$ & $5(9.6)$ & $0.81(0.3 \mid 2.09)$ \\
\hline No violencia & $1031(87.2)$ & $146(12.3)$ & 1.0 Referencia \\
\hline
\end{tabular}


estuvo asociada con un mayor reporte de este trastorno. Por ejemplo se observó que la dispareunia fue reportada 9.4 veces más en mujeres con dolor crónico de la pelvis (IC 95\% 6.21-14.26), comparado con mujeres que reportaban no tener esta condición. El análisis bivariado muestra que tener un diagnóstico de infecciones urinarias crónicas y trastornos intestinales (colitis, colitis ulcerativas y enfermedad de Crohn) también incrementa la probabilidad de reporte de dispareunia. La violencia sexual en todas las modalidades analizadas indica la existencia de una asociación estadística entre dichas variables y dicho trastorno.

El tiempo promedio que las mujeres han experimentado dispareunia, la edad promedio en que dicho trastorno se manifestó por primera vez, la percepción de severidad del mismo y el uso de servicios médicos se presentan en el cuadro III. El tiempo promedio reportado con dicho trastorno fue de 6 años, mientras que la edad promedio de inicio de los síntomas de dispareunia fue a los 29 años. La dispareunia fue reportada como un evento doloroso de intensidad moderada o severa en $46 \%$ de las mujeres con este trastorno. Las mujeres reportaron cambios en la frecuencia de la actividad sexual. Al respecto, $41 \%$ de las mujeres con esta condición señaló haber reducido el número de relaciones sexuales que ellas hubieran deseado tener. Sólo una de cada 5 mujeres reportó haber hablado con su médico respecto a este trastorno los 12 meses previos a la encuesta.

El cuadro IV presenta los resultados de la regresión logística múltiple. Se observó una interacción estadística entre la presencia de dispareunia y las variables de edad y antecedentes de enfermedades de transmisión sexual y / o enfermedad inflamatoria pélvica (ETS-EIP),

Cuadro III

TIEMPO Y EDAD DE EXPERIMENTAR DISPAREUNIA, PERCEPCIÓN DE SEVERIDAD, CAMBIO EN LA FRECUENCIA DE LA ACTIVIDAD SEXUAL Y CONTACTO CON LOS SERVICIOS DE SALUD. $\mathrm{N}=\mid 46$. ENCUESTA SOBRE MORBILIDAD GINECOLÓGICA Y CALIDAD de vida en mujeres en edAd Reproductiva. Hermosillo, SONORA, 2005

Dispareunia

\begin{tabular}{lc} 
Tiempo promedio experimentando dispareunia (media, DS) & $5.8 \pm 6.3$ \\
\hline N/R & I \\
\hline Edad media en la que se experimenta dispareunia (media, DS) & $29.5 \pm 8.55$ \\
\hline N/R & I \\
\hline Dolor durante el coito se percibe como moderado o severo (\%) & $67(45.9)$ \\
\hline Modificó la frecuencia de la actividad sexual debido al dolor (\%) & $60(41.0)$ \\
\hline
\end{tabular}

Habló con un médico sobre sus síntomas durante el pasado año (\%) 33 (22.6)
Cuadro IV

RAZÓn de MOMIOS aJUSTADAS DE LA ASOCIACIÓN ENTRE DISPAREUNIA Y ESTATUS OCUPACIONAL, MORBILIDAD Y VIOLENCIA SEXUAL EN POBLACIÓN FEMENINA.

ENCUESTA SOBRE MORBILIDAD GINECOLÓGICA Y CALIDAD DE VIDA EN MUJERES EN EDAD REPRODUCTIVA. Hermosillo, Sonora, 2005

\begin{tabular}{ll} 
Características & RMA (IC 95\%) \\
Ocupación & \\
Trabaja & 1.58 (I.08 2.30) \\
\hline Hogar/otro & 1.0 Referencia
\end{tabular}

\begin{tabular}{|c|c|}
\hline \multicolumn{2}{|c|}{ Edad/Antecedentes de ETS-EIP } \\
\hline 25-34 años/ETS-EIP & $5.67(3.00$ I0.73) \\
\hline 25-34 años/No ETS-EIP & $1.71(1.132 .60)$ \\
\hline 35-54 años /ETS-EIP & $0.97(0.48$ I.96) \\
\hline 35-54 años/ No ETS-EIP & I.0 Referencia \\
\hline
\end{tabular}

Infección urinaria crónica

$\begin{array}{ll}\text { Sí } & 1.83(1.242 .70) \\ \text { No } & 1.0 \text { Referencia }\end{array}$

Colitis ulcerativa y otros trastornos intestinales

\begin{tabular}{ll} 
Sí & $1.62(1.102 .40)$ \\
\hline No & 1.0 Referencia
\end{tabular}

Violencia sexual de toda la vida

\begin{tabular}{ll} 
Sólo antes de los I5 años & $3.72($ I.90 7.27) \\
\hline Sólo a partir de los I5 años & $1.99($ I.12 3.53) \\
\hline Antes y después de los I5 años & $2.92($ I.33 6.4I) \\
\hline No violencia & I.0 Referencia
\end{tabular}

lo anterior después de ajustar por ocupación, morbilidad y violencia sexual. La edad por sí sola aparece también como un factor asociado con dispareunia dado que el grupo de mujeres adultas jóvenes sin antecedentes de ETS-PID tendieron a reportar con mayor frecuencia esta condición. La violencia sexual estuvo asociada con el reporte de dispareunia. Se observa un incremento en el reporte de dispareunia en mujeres que fueron violentadas sexualmente de niñas o durante diferentes etapas de su vida y/o por diferentes perpetradores. Por ejemplo, en comparación con las mujeres que no informaron violencia, aquellas que reportaron violencia sexual antes de los 15 años fueron 3.7 veces más propensas a relatar esta condición. Por otra parte, la dispareunia fue 2.9 veces más común (IC 95\% 1.33-6.41) en mujeres que reportaron violencia sexual en diferentes etapas de su ciclo de vida. Una asociación significativa entre 
dispareunia y condiciones genitourinarias y trastornos intestinales también fue observada. La dispareunia fue 53\% más común en mujeres que reportaron haber trabajado la semana previa a la encuesta.

\section{Discusión}

De acuerdo a nuestros datos, la dispareunia femenina es un trastorno sexual común en mujeres mexicanas en edad reproductiva. Como se pudo observar, en el área urbana de la ciudad de Hermosillo, Sonora una de cada 8 mujeres entre 25-54 años de edad que reportó haber tenido una pareja sexual los 12 meses previos a la encuesta, manifestó haber tenido dolor pélvico durante el coito vaginal o 24 horas después de dicho evento. Asimismo, una de cada 17 mujeres calificó tener relaciones sexuales con dolor moderado o severo, en tanto una de cada 19 mujeres señaló cambios en su conducta sexual como resultado de dicho trastorno sexual. Las mujeres con dispareunia informaron tener en promedio seis años padeciendo dicha condición. La prevalencia de dispareunia reportada en este estudio se encuentra dentro del rango señalado en estudios previos. ${ }^{8}$

Los factores de riesgo asociados con la dispareunia femenina en población mexicana son consistentes con hallazgos documentados previamente. En este estudio, la presencia de morbilidades concurrentes y los antecedentes de violencia sexual estuvieron asociados con este trastorno. ${ }^{8}$

Dentro de nuestros hallazgos, es importante resaltar el rol que tiene la violencia sexual como factor de riesgo para la salud sexual. ${ }^{15}$ Dicha asociación identifica que un número importante de mujeres son incapaces de decidir cuándo, cómo y con quién relacionarse sexualmente durante diferentes etapas de su vida. Esta situación es prevalente aun en mujeres que viven dentro de una relación conyugal estable, como es el caso de las mujeres casadas o unidas. La violencia sexual es un fenómeno que acompaña el inicio de la actividad sexual para algunas mujeres. Como se pudo observar en este estudio, mujeres que experimentaron violencia sexual durante la niñez y aquéllas que fueron violentadas por diferentes perpetradores en diferentes etapas de su vida fueron más proclives a reportar dispareunia. ${ }^{16} \mathrm{La}$ violencia sexual contra mujeres dentro del matrimonio o dentro de uniones consensuales parece ser un evento común. ${ }^{17-19}$ Los datos de este estudio muestran que $8 \%$ de las mujeres que han vivido o cuentan con una pareja sexual han sido forzadas a tener relaciones sexuales con su cónyuge cuando ellas no lo deseaban.

En este estudio 13\% de las mujeres reportaron tener antecedentes de ETS y / o EIP. Nuestros datos muestran un incremento en la probabilidad del reporte de dis- pareunia en mujeres adultas jóvenes (25-34 años) con antecedentes de ETS y/o EIP. Tanto la edad como los antecedentes de ETS y EIP han sido reportados como factores de riesgo de dispareunia en otros estudios. ${ }^{8}$

Es claro que la presencia de trastornos sexuales en uno de los cónyuges interrumpe y modifica el intercambio sexual de las parejas y por lo tanto podría afectar la cohesión y estabilidad de las mismas. De acuerdo a nuestros datos, una de cada 19 mujeres en edades de 25 a 54 años ha reducido la frecuencia en el número de relaciones sexuales que ella hubiera deseado tener como resultado de la dispareunia. Este escenario sugiere la existencia de "una demanda insatisfecha de relaciones sexuales" en la población femenina mexicana asociadas con este trastorno sexual. Existe evidencia de que algunos trastornos como la dispareunia, el vaginismo y la vulvodinia afectan negativamente la vida sexual de las mujeres. ${ }^{20-22}$

Una de las recomendaciones de la OMS en materia de salud sexual y reproductiva es que los programas en este campo garanticen el ejercicio pleno de la sexualidad de la población. ${ }^{23}$ Para el logro de lo anterior se necesita identificar las barreras sociales y fisiológicas que lo impiden. Con la prevención de trastornos sexuales como la dispareunia se cubriría la "demanda insatisfecha de relaciones sexuales" asociadas a dicho trastorno, lo que permitiría cumplir con una de las premisas descrita en la definición de salud sexual de la OMS, donde se establece el derecho de los individuos a ejercer una sexualidad libre de trastornos físicos y enfermedad. ${ }^{23}$

Adicionalmente, los programas de salud sexual y reproductiva deben promover el derecho de los individuos a disfrutar de una vida sexual en concordancia con su ética personal y social. ${ }^{23}$ De acuerdo con esta definición, el ejercicio de la sexualidad debe darse en un ambiente libre de miedo, vergüenza, culpa y fuera de creencias falsas acerca de la sexualidad. ${ }^{23}$ Los datos de este estudio muestran que la violencia sexual a mujeres impide que estas premisas se cumplan cabalmente. La conexión entre dispareunia y violencia sexual demanda la evaluación sistemática de ambos eventos, principalmente la evaluación de la violencia en el contexto clínico a nivel de prevención primaria y secundaria. En el contexto de la evaluación clínica de los trastornos sexuales y/o pélvicos, hay evidencias de la eficacia del autorreporte de violencia sexual y trastornos sexuales por parte de las pacientes cuando hay un autollenado de la historia clínica por parte de las mismas. ${ }^{24}$

La presencia de trastornos ginecológicos concurrentes a la dispareunia demanda que los programas de salud reproductiva giren no solamente en torno a enfermedades que ponen en riesgo la vida de las mu- 
jeres. ${ }^{1,25}$ Es necesario evaluar de manera más sistematizada indicadores de morbilidad ginecológica dado el posible efecto que éstos tienen en la calidad de vida de las mujeres. En el contexto mexicano se ha observado que morbilidades como la dispareunia, el dolor pélvico crónico, la dismenorrea y la incontinencia urinaria están afectando la calidad de vida de las mujeres en edad reproductiva dado que son una fuente de dolor, producen ansiedad y modifican la conducta sexual y social de las mismas. ${ }^{7}$ En este estudio se observó una asociación entre dispareunia y la presencia de morbilidades como infecciones crónicas del tracto urinario ${ }^{11,26} \mathrm{y}$ enfermedades inflamatorias del intestino. ${ }^{27} \mathrm{Lo}$ anterior describe un escenario de causas fisiológicas múltiples que deben ser simultáneamente evaluadas por los proveedores de salud.

La aparente falta de interés en los trastornos sexuales de parte de los programas de salud reproductiva opera como una barrera para que las mujeres hablen abiertamente de su sexualidad con los proveedores de salud. La sexualidad sigue siendo un tema tabú en México. ${ }^{28}$ Lo anterior explica parcialmente por qué los programas de salud reproductiva se enfocan sólo en las "consecuencias" que acompañan el ejercicio de la sexualidad, como el embarazo y las enfermedades de transmisión sexual, y no promueven el tamizaje o escrutinio de los trastornos sexuales. Según Dávila ${ }^{28}$ el silencio o la falta de discusión sobre la sexualidad atenta contra la salud de las mujeres ya que este hecho restringe el acceso a información en salud sexual e incrementa el riesgo de las mujeres a embarazos no deseados y enfermedades de trasmisión sexual..$^{28}$ Ante esta situación es necesario que los proveedores de salud tengan una actitud más proactiva en la evaluación de los trastornos sexuales.

Nuestros resultados tienen algunas limitaciones y fortalezas. La evaluación de los trastornos ginecológicos, incluyendo la dispareunia, son resultado del autorreporte de las mujeres y no se cuenta con evaluaciones clínicas que validen dichos reportes. Sin embargo, se alcanzaron tasas de respuesta muy altas en la evaluación de los problemas de salud y se utilizaron cuestionarios validados para el análisis de estos problemas, además de que los hallazgos son consistentes con los resultados de otros estudios.

Este estudio hace aportaciones relevantes en el contexto de la morbilidad ginecológica. Hasta donde sabemos, este estudio es el primero en reportar información poblacional sobre la prevalencia de la dispareunia femenina y algunos de sus factores asociados en el contexto urbano del noroeste de México.

\section{Referencias}

I. Cottingham J, Myntti C. Reproductive Health: Conceptual Mapping and Evidence. In: Sen G, George A, Ostlin P, eds. Engendering International Health:The Challenge of Equity. Massachusetts: Institute of Technology, 2002:85-109.

2. Salles V,Tuirán R. El discurso de la salud reproductiva: ¿un nuevo dogma? In: Stern C, Figueroa JG, eds. Sexualidad y salud reproductiva. Avances y retos para la investigación. Ciudad de México: El Colegio de México, 2001:93-113.

3. CONAPO. Cuadernos de Salud Reproductiva, República Mexicana. DF: Consejo Nacional de Población, 2000.

4. Rojas-Poceros G, Bustos López HH, Oviedo-Ortega JG, GonzálezCofrades J, Ibarrola-Buen Abad E. Perspectiva y alcances de la endoscopía ginecológica en el hospital $A B C$ : revisión de 10 años. Perinatol Reprod Human 200I;I5(4):229-237.

5. Sánchez-Bravo C, Carreño-Meléndez J, Martínez-Ramírez S, GómezLópez ME. Disfunciones sexuales femeninas y masculinas: comparación de género en una muestra de la Ciudad de México. Salud Mental 2005;28(4):74-80.

6. Sánchez-Bravo C, Morales-Carmona F, Carreño-Meléndez J, MartínezRamírez S. Disfunción sexual femenina: su relación con el rol de género y la asertividad. Perinatología y reproducción humana 2005;19(3-4):152-160. 7. García-Pérez MH.The Impact of Gynecological Morbidity on Women's Quality of Life in Northern Region of Mexico (Tesis).Ann Arbor: University of Michigan, 2006:I46.

8. Latthe P, Mignini L, Gray R, Hills R, Khan K. Factors predisposing women to chronic pelvic pain: systematic review. Bmj 2006;332(7544):749-755.

9. Rosen RC. Prevalence and risk factors of sexual dysfunction in men and women. Curr Psychiatry Rep 2000;2(3):189-195.

10. Heim LJ. Evaluation and differential diagnosis of dyspareunia. Am Fam Physician 2001;63(8):I535-1544.

II. Laumann EO, Paik A, Rosen RC. Sexual dysfunction in the United States: prevalence and predictors. JAMA 1999;28I (6):537-544.

12. Zondervan KT,Yudkin PL, Vessey MP, Jenkinson CP, Dawes MG, Barlow $\mathrm{DH}$, et al. Chronic pelvic pain in the community--symptoms, investigations, and diagnoses. Am J Obstet Gynecol 200 I; I84(6): I I49-1 I 55.

13. Osborn M, Hawton K, Gath D. Sexual dysfunction among middle aged women in the community. Br Med J (Clin Res Ed) 1988;296(6627):959-962. 14. WHO.WHO Multy-Country Study on Women's Health and Domestic Violence Against Women. Geneva:World Health Organization, 2003.

15. Golding JM,Wilsnack SC, Learman LA. Prevalence of sexual assault history among women with common gynecologic symptoms. Am J Obstet Gynecol 1998; 179(4):1013-1019.

16. Chapman JD.A longitudinal study of sexuality and gynecologic health in abused women. J Am Osteopath Assoc 1989;89(5):619-624.

17. Rivera-Rivera L,Allen B, Chavez-Ayala R,Avila-Burgos L. [Physical and sexual abuse during childhood and revictimization during adulthood in Mexican women]. Salud Publica Mex 2006;48 Suppl 2:S268-S278. 18. Silverman JG, Decker MR, Saggurti N, Balaiah D, Raj A. Intimate partner violence and HIV infection among married Indian women. Jama 2008;300(6):703-710.

19. Weiss HA, Patel V,West B, Peeling RW, Kirkwood BR, Mabey D. Spousal sexual violence and poverty are risk factors for sexually transmitted infections in women: a longitudinal study of women in Goa, India. Sex Transm Infect 2008;84(2): 133-139.

20. Plante AF, Kamm MA. Life events in patients with vulvodynia. Bjog 2008; II5(4):509-5I4. 
21. Jamieson DJ, Steege JF.The prevalence of dysmenorrhea, dyspareunia, pelvic pain, and irritable bowel syndrome in primary care practices. Obstet Gynecol 1996;87(I):55-58.

22. Mathias S, Kuppermann M, Liberman R, Lipschutz R, Steege J. Chronic pelvic pain: prevalence, health-related quality of life, and economic correlates. Obstet Gynecol 1996;87(3):32I-327.

23. WHO. Education and treatment in human sexuality: the training of health professionals. Geneva:World Health Organization; 1975. Report No.: 572 .

24. Elzevier HW,Voorham-van der Zalm PJ, Pelger RC. How reliable is a self-administered questionnaire in detecting sexual abuse: a retrospective study in patients with pelvic-floor complaints and a review of literature. J Sex Med 2007;4(4 Pt I):956-963.
25. Glasier A, Gulmezoglu AM, Schmid GP, Moreno CG, Van Look PF. Sexual and reproductive health: a matter of life and death. Lancet 2006;368(9547):1595-1607.

26. Salonia A, Zanni G, Nappi RE, Briganti A, Deho F, Fabbri F, et al. Sexual dysfunction is common in women with lower urinary tract symptoms and urinary incontinence: results of a cross-sectional study. Eur Urol 2004;45(5):642-648.

27. Zondervan KT,Yudkin PL,Vessey MP, Jenkinson CP, Dawes MG, Barlow $\mathrm{DH}$, et al. The community prevalence of chronic pelvic pain in women and associated illness behaviour. Br J Gen Pract 200 I;5I (468):54I-547.

28. Davila YR. The social construction and conceptualization of sexual health among Mexican American women. Res Theory Nurs Pract 2005; 19(4):357-368. 